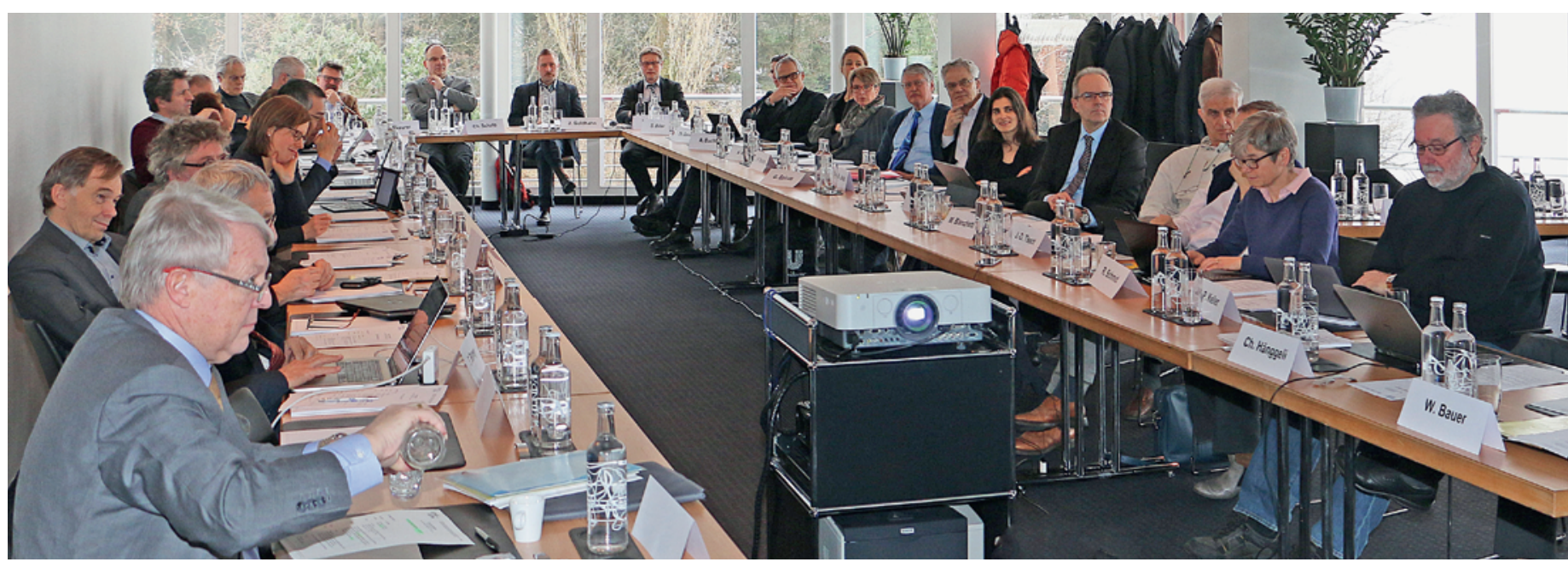

Die Journée de réflexion bringt Expertinnen und Experten auf dem Gebiet der ärztlichen Bildung zusammen.

\title{
Anstösse vermitteln, die zu Ergebnissen führen
}

\section{Bruno Kesseli}

Dr. med. et lic. phil., Chefredaktor

Die traditionelle Journée de réflexion von SIWF und "Collège des Doyens» bot auch in diesem Jahr anregende Referate und gab Experten aus dem Bereich der ärztlichen Aus- und Weiterbildung Gelegenheit zu einem intensiven Austausch.

Die Gastgeber waren sich einig. Sowohl Henri Bounameaux, Präsident des "Collège des Doyens», als auch Werner Bauer, Präsident des SIWF, unterstrichen in ihren Begrüssungsansprachen die Bedeutung der Journée de réflexion als Informations- und Diskussionsforum für Keyplayers auf dem Gebiet der ärztlichen Bildung. Ein Blick auf die Teilnehmerliste bestätigte, dass diese in beeindruckender Dichte anwesend waren.

\section{Mehr als ein zwangloser Austausch}

Werner Bauer betonte indes, dass es nicht nur darum gehe, sich zwanglos auszutauschen. Hinter der Veranstaltung stehe auch der Anspruch, Anstösse zu vermitteln, die letztlich zu konkreten Ergebnissen führten. Henri Bounameaux konnte dazu gleich mit einem Beispiel aufwarten. Im Rahmen der "Journée 2016» hatte das «Collège des Doyens» eine Stellungnahme zur Organisation des Medizinstudiums verabschiedet, die auch in der SÄZ publiziert wurde [1] und die Diskussion um Veränderungen in der Ärzteausbildung wahrnehmbar beeinflusst habe. Bounameaux wies auch auf verschiedene weitere Engagements des «Collège des Doyens» hin, etwa in Arbeitsgruppen des Bundesamts für Gesundheit (BAG) und der Schweizerischen Akademie der Medizinischen Wissenschaften SAMW.

\section{Journée de réflexion}

Die Journée de réflexion ist eine seit Jahren etablierte Einladungsveranstaltung zu Fragen der ärztlichen Bildung. Organisiert wird sie vom Schweizerischen Institut für ärztliche Weiter- und Fortbildung SIWF sowie dem Kollegium der Dekane der Schweizerischen Medizinischen Fakultäten. Die diesjährige Journée de réflexion fand am 20./21. Januar in Luzern statt. 


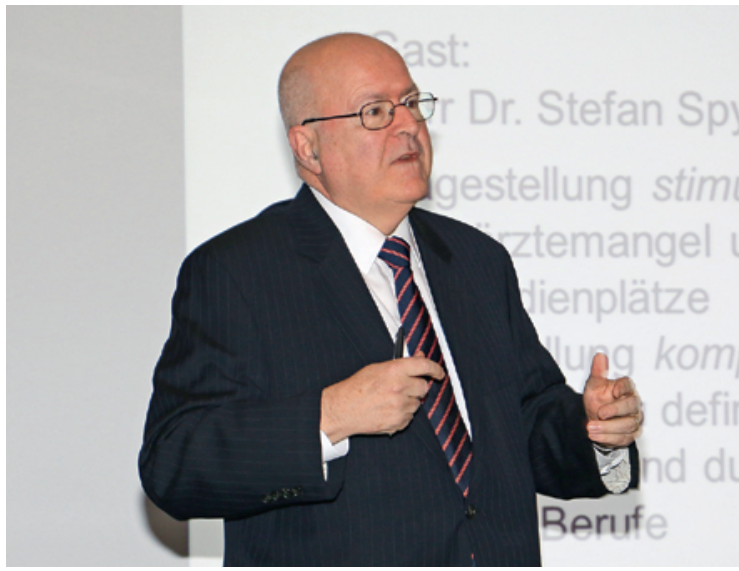

Co-Gastgeber Werner Bauer begrüsste die Anwesenden und umriss die Ziele der Veranstaltung.

\section{Optimale medizinische Versorgung der Bevölkerung}

Nachdem SIWF-Geschäftsführer Christoph Hänggeli die vielfältigen Aufgaben und Aktivitäten des Instituts in einem dichten Info-Flash zusammengefasst hatte, referierte Mauro Poggia zum Thema Couverture médicale optimale de la population suisse: problèmes et pistes de solution. Der Gesundheitsdirektor des Kantons Genf erinnerte zunächst an Grundsätzliches, das für die medizinische Versorgung der Schweiz allerdings nicht trivial ist. Als Konföderation verfüge die Schweiz über ein besonders komplexes Gesundheitssystem, das bei vielen unbestrittenen Vorzügen vor allem in Bezug auf die Finanzierung langsam an seine Grenzen stosse. Von den involvierten Akteuren hätten lediglich die Kantone ein echtes Interesse daran, die Gesundheitskosten zu limitieren. Generell sei die Tendenz der Stakeholder zu beobachten, sich aus der Verantwortung zu verab- schieden («Déresponsabilisation»). Beim Bund in seiner Rolle als Gesetzgeber ortet Poggia eine gewisse Passivität, in Bezug auf die Datenlage stellt er Intransparenz fest und die Steuerung des Systems bezeichnet er als ineffizient. Als Beispiele für die «Déresponsabilisation» nannte er die Tendenz zur Mengenausweitung durch die Leistungserbringer, den insuffizienten Risikoausgleich der Versicherer, den Überkonsum von Leistungen durch die Prämienzahler und die Idealisierung des Wettbewerbsmodells durch Bund und Kantone.

\section{Ausgleichskasse als Lösung?}

Vor dem Hintergrund bekannter zukünftiger Herausforderungen wie der Zunahme polymorbider alter $\mathrm{Pa}$ tienten sei eine Reform des Systems unumgänglich. Poggia hat auch klare Vorstellungen darüber, wie diese Reform auszusehen hätte. Ihm schwebt ein Modell vor, das den Kantonen die Möglichkeit bietet, eine "Ausgleichskasse» einzuführen. Diese würde die Prämien festlegen und einkassieren, während die Krankenkassen weiterhin für die Kontrolle der Rechnungen und die Betreuung der Versicherten zuständig wären. Erwünschte Folge wäre die Vereinheitlichung der Prämien innerhalb der einzelnen Kantone, wodurch die Jagd auf gute Risiken entfiele. Die Verfechter dieses Modells versprechen sich davon eine stabilisierende Wirkung auf Prämien und Tarife. Naturgemäss ist umstritten, ob diese Vorstellung realistisch ist, wie die medial geführten Diskussionen der Idee zeigen, die im Sommer mit einer eidgenössischen Volksinitiative konkretisiert werden soll.

Wie aus der anschliessende Diskussion hervorging, befürwortet Poggia auch weitere Massnahmen wie die örtliche Steuerung der Niederlassung. Im heutigen

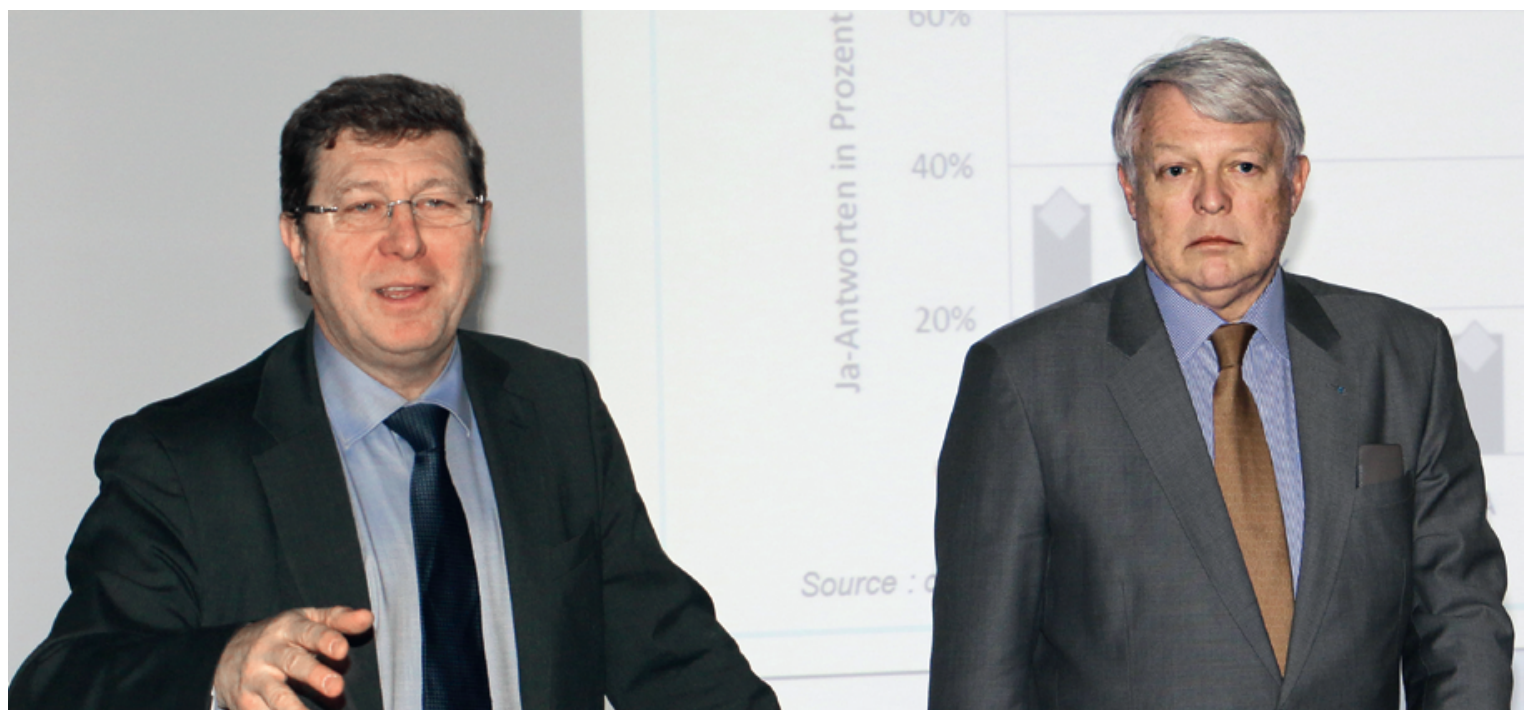

Referent Mauro Poggia und Co-Gastgeber Henri Bounameaux. 
Umfeld könne der Arztberuf nicht total liberal sein, meinte er. Werner Bauer warf die Frage nach den Implikationen solcher kostenzentrierten Betrachtungen auf die ärztliche Bildung auf, und Henri Bounameaux wies zusätzlich darauf hin, dass die zahlreichen koordinativen Aufgaben der Grundversorger generell schlecht abgegolten seien. Poggia betonte, für diese Bereiche müsste ausreichend Mittel bereitgestellt werden, da sonst wesentliche Dimensionen der Medizin verloren gingen. Aber die Ärzte müssten auch offen sein, neue unterstützende Berufe zu akzeptieren, gerade im Bereich der Koordination von Gesundheitsleistungen.

\section{Steuerung der ärztlichen Versorgung}

Die Steuerung der ärztlichen Workforce war Thema des zweiten Referats der Veranstaltung, das von BAG-Vizedirektor Stefan Spycher gehalten wurde. Werner Bauer vertrat in der Einleitung den Standpunkt, dass sich die Ärzteschaft aktiv mit diesem Thema auseinandersetzen sollte und stellte das «Modell Holland» vor, das zurzeit von vielen als Beispiel für die Schweiz gesehen werde. Es hat zum Ziel, basierend auf dem aktuellen Bestand und den prognostizierten Entwicklungen den zukünftigen Bedarf an Ärztinnen und Ärzten abzuschätzen. Dabei wird eine ganze Reihe von Faktoren berücksichtigt, darunter demographische, epidemiologische und technologische.

Spycher identifizierte fünf grosse Probleme im Zusammenhang mit der ärztlichen Versorgung in der Schweiz:

- Zu wenig Studierende / hohe Auslandsabhängigkeit

- Hohes Alter beim Abschluss der Weiterbildung

- Das Angebot der Weiterbildungsstellen entspricht nicht dem Bedarf

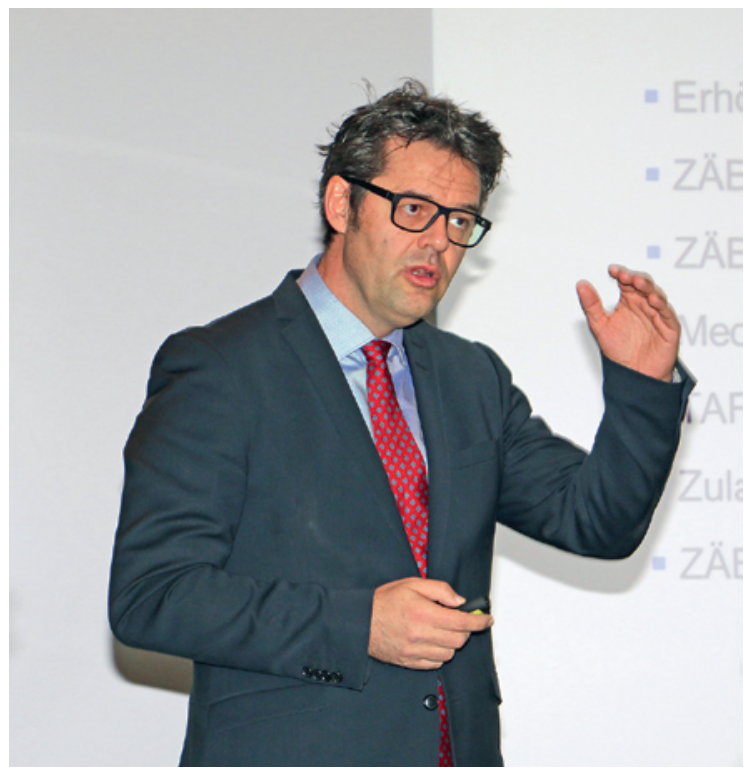

Stefan Spycher referierte zu Steuerungsfragen.
- Ungesteuerte Niederlassung im ambulanten Bereich - vor allem von ausländischen Ärzten/Ärztinnen - kann die Kosten ohne klaren Zusatznutzen für die Patienten/Patientinnen erhöhen

- Trotz genügend Ärzten: Schwierigkeiten der Übergabe von Praxen in ländlichen Regionen

Der Bund hat bereits verschiedene Initiativen lanciert, die zur Problemlösung beitragen sollen. Spycher nannte etwa die Erhöhung der Anzahl Studierender und verschiedene Arbeitsgruppen der Plattform «Zukunft ärztliche Bildung», darunter eine, die sich spezifisch mit Fragen der Koordination der ärztlichen Weiterbildung befasst. Auch die Zulassungssteuerung und die Reduktion von Fehlanreizen via TARMED-Revision zählen für ihn zu diesem Paket. Sein Fazit lautete, dass die Workforce-Planung einen Beitrag zur Lösung des Problems darstellen kann, dass aber an verschiedenen Stellschrauben anzusetzen ist, wenn die hohe Qualität der Aus- und Weiterbildung sowie der ärztlichen Versorgung in der Schweiz erhalten bleiben oder sogar verbessert werden soll.

\section{Keine Patentrezepte}

Die nachfolgende Diskussion machte deutlich, dass niemand ein Patentrezept aus dem Ärmel schütteln kann, mit dem sich die bestehenden und die sich abzeichnenden Herausforderungen integral bewältigen lassen. Werner Bauer wies auf einen Punkt hin, der nach seiner Einschätzung bei der Entwicklung von Lösungsansätzen zentral sei. Das Berufsbild des Hausarztes werde sich verändern, zeigte er sich überzeugt, und dies müsse in den Überlegungen der involvierten Akteure unbedingt berücksichtigt werden. Spycher stimmte ihm zu und versicherte, dass diese Frage in verschiedenen Expertengremien tatsächlich thematisiert werde. Breiten Raum nahm die Erörterung von Steuerungsfragen ein. Johann Steurer, Direktor des Horten-Zentrums für praxisorientierte Forschung und Wissenstransfer der Universität Zürich, plädierte wie bereits bei früheren Gelegenheiten [2] dafür, mit der Spezialisierung bereits nach dem Bachelorabschluss im Medizinstudium zu beginnen. Von verschiedenen Votanten wurde betont, dass Geldfragen im Zusammenhang mit der Steuerung sehr wohl eine Rolle spielten. Rainer Weber, Dekan der Medizinischen Fakultät der Universität Zürich, brachte einen weiteren Aspekt ein. Auch ein Zuviel an Ärzten sollte thematisiert werden, meinte er. In gewissen Fachrichtungen sei dies eindeutig der Fall. Das Problem werde zusätzlich dadurch verschärft, dass wir alle Fachärzte aus dem EU-Raum zulassen müssten - auch solche, die allenfalls ungenügend aus- und weitergebildet sind. 


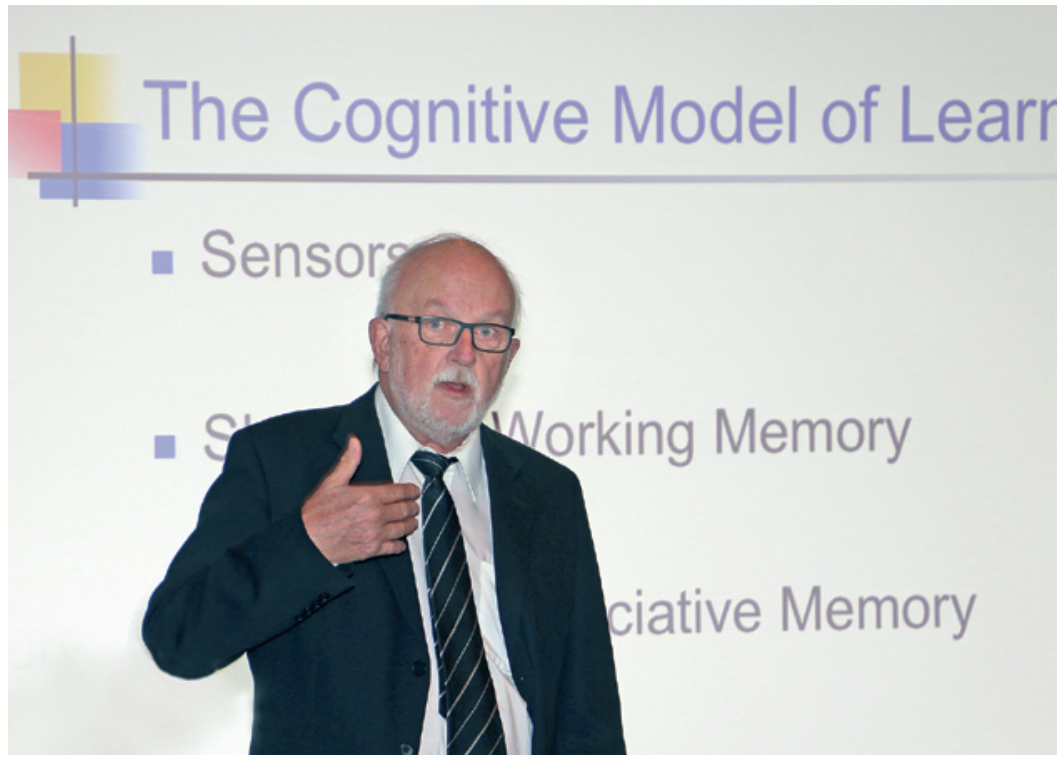

Lernprozesse aktiv gestalten: Geoff Norman.

\section{Das Hirn funktioniert assoziativ}

Faszinierende Einblicke in die Art und Weise, wie Menschen lernen, vermittelte Geoff Norman in seinem Referat How People learn and how this affects teaching and curriculum. Der Nuklearphysiker, Psychologe und Professor emeritus für klinische Epidemiologie und Biostatistik der McMaster University, Kanada, befasst sich in seiner Arbeit intensiv mit der Medical Education, deren Instrumente er wissenschaftlich erforschte und zu deren Weiterentwicklung er wichtige Beiträge leistete. An verschiedenen Beispielen machte Norman deutlich, dass die Metapher vom menschlichen Hirn als Computer den Kern nicht erfasst. Die Funktionsweise des Hirns unterscheidet sich in wesentlichen Aspekten fundamental vom Computer. Es ist viel langsamer und seine Datenverarbeitungskapazität sowie sein Arbeitsspeicher sind viel kleiner. Dagegen ist die Speicherkapazität des Hirns fast unvorstellbar gross. Unter diesen Voraussetzungen ist es erstaunlich, dass wir überhaupt in der Lage sind, bei gewissen Fragestellungen ähnlich gute Resultate zu erzielen wie ein Computer. Wenn wir beispielsweise nach der Hauptstadt von Frankreich gefragt werden, wissen wir die richtige Antwort sofort, und wir wissen, dass wir sie wissen. Werden wir dagegen nach der Hauptstadt von Tadschikistan gefragt, wissen wir ebenso rasch, dass wir die Antwort nicht wissen (zumindest viele von uns). Solche Resultate sind nur möglich, weil das Hirn assoziativ auf das Gedächtnis zugreift und dieses nicht sequentiell abfragt wie ein Computer. Umgekehrt werden bei Lernvorgängen nicht isolierte Informationen abgespeichert, stehendem Wissen verknüpft. Die Zuschreibung von Bedeutung ist somit für den Lernvorgang zentral. Beispielsweise sind Schachexperten im Vergleich zu Hobbyspielern in der Lage, sich ein Vielfaches an realen Spielpositionen zu merken, wenn sie diese während fünf Sekunden sehen. Handelt es sich dagegen um zufällige, "sinnlose» Anordnungen der Figuren auf dem Brett, schneiden die Experten nicht besser ab als die Hobbyspieler, da sie das Bild nicht mit ihrem Vorwissen verknüpfen können.

Solche Erkenntnisse sind auch für die ärztliche Bildung wichtig. Lernprozesse sollten aktiv gestaltet werden, Prinzipien sollten wenn möglich eingebettet in konkrete Problemstellungen vermittelt werden und zu ihrem Verständnis sollte auf Analogien aus dem Alltag zurückgegriffen werden. Dass eine solche Art der Wissensvermittlung zu besseren Resultaten führt als "sequentielles Lernen" - erst das Prinzip erklären, dann Beispiele dafür geben - belegte Norman anhand verschiedener Studien.

\section{Von der Reflexion zur Aktion}

Im abschliessenden Themenblock zu "offenen Fragen der ärztlichen Bildung» ergab sich eine intensive Diskussion, aus denen Werner Bauer unter dem Schlagwort Von der Reflexion zur Aktion eine mögliche To-do-List ableitete. Vorsichtshalber versah er einige der entsprechenden Punkte mit einem Fragezeichen. Angeregt wurde etwa, den Stellenwert des Teachings in den Spitälern zu heben, die Anzahl Weiterbildungsstellen zu hinterfragen, die Lernzielkataloge zu überdenken oder die Weiterbildung vermehrt in Netzwerken zu strukturieren. Ebenfalls auf die Liste schafften es die Frage, ob (und welche) ärztlichen Arbeiten durch andere Fachpersonen übernommen werden könnten, die Sicherstellung der Finanzierung der Weiterbildung, das Zusammengehen von Weiterbildung und wissenschaftlicher Tätigkeit und die Entwicklung von Tracks im Studium.

Die anwesenden Expertinnen und Experten konnten den Heimweg somit mit einem gut gefüllten «Ideenrucksack» antreten. Man darf gespannt sein, ob an der nächsten Journée zu einzelnen Fragen und Problemen bereits konkrete Lösungen präsentiert werden.

\section{Literatur}

1 Bounameaux H, Eggli P, Gasser T, Tissot JD, Weber R. Statement regarding medical education in Switzerland. Schweiz Ärztezeitung. 2016;97(12/13):461-2.

2 Kesseli B. Zukünftigen Herausforderungen wirkungsvoll begegnen. Schweiz Ärztezeitung. 2015;96(45):1638-41.

Bildnachweis

Fotos Bruno Kesseli 